\title{
PRODUCT SPACES IN LOCALES
}

\author{
JOHN ISBELL
}

\begin{abstract}
The embedding of sober spaces in locales preserves products $X \times Y$ where $X$ is quasi-locally compact. A completely regular space $X$ has this preservation property, for all $Y$, if and only if $X$ is a complemented sublocale of a compact space. Equivalently, every closed subset of $X$ is locally compact somewhere.
\end{abstract}

This paper assumes such familiarity with locales as can be gotten from the first nine pages of [4]. There will be a couple of references to later results from [4], but the first nine pages (through 1.5) establish the conceptual apparatus.

The referee suggested that "a brief description of the product locale of two spaces ... would be helpful". Yes, indeed. The existence of these products, i.e. of coproducts in the dual category, was an unsolved problem for some time until Benabou proved existence [1]. But it happens that, after the first version of this paper was written, D. Wigner sent me his beautiful brief description (to be published). Recall, the dual object of a locale $X$ is the complete Heyting algebra $T(X)$ of open parts of $X$. If $X$ is a (sober) space, $T(X)$ is just its topology.

Wigner gets the product $X \times Y$ from Galois connections between $T(X)$ and $T(Y)$. Note, this can be skipped; Wigner's theorem will not be used below. But a Galois connection between two partially ordered sets $P, Q$, consists of two functions (order-reversing) $f: P \rightarrow Q, g: Q \rightarrow P$, such that $y<f(x)$ if and only if $x<g(y)$. These connections are partially ordered, $(f, g)<\left(f^{\prime}, g^{\prime}\right)$ if $f<f^{\prime}$ pointwise. (Equivalently, $g \leqslant g^{\prime}$.) It was known [5] that the Galois connections between two complete Heyting algebras $A, B$, so ordered, form a complete Heyting algebra $A \otimes B$. Wigner showed that $T(X) \otimes T(Y)$ is $T(X \times Y)$.

Turning toward proofs, we need to name the spaces $X$ whose topology is a continuous lattice. Various names have been used (e.g. in [4]), but A. J. Ward's quasi-locally compact seems to be prevailing. The defining (lattice-theoretic) property: each open set $U$ is the union of those open sets $B$ such that every open cover of $U$ has a finite subcollection covering $B$, i.e. $B$ is bounded in $U$.

Actually, to justify identifying topological spaces $X$ with the corresponding locales, we need to assume all spaces are sober. (It is easy to check that the obvious interpretations of the theorems below for nonsober spaces remain true-vacuously for Hausdorff spaces, which are sober.) Then one should note another nice, rather new theorem, which will not be used: sober quasi-locally compact spaces are locally quasi-compact [3].

Received by the editors September 27, 1979 and, in revised form, November 19, 1979. 1980 Mathematics Subject Classification. Primary 54H99; Secondary 54B10. Key words and phrases. Locale, quasi-locally compact. 
To finish introducing: Theorem 1 is of interest mainly for the proof, which can lead on to Theorem 4. Theorem 1 for Hausdorff spaces is known [2].

TheOrem 1. A locale $X \times Y$ is a space if $X$ is a quasi-locally compact space and $Y$ is a space.

Proof. Since open rectangles $U \times V$ form a basis, it suffices to show that $U \times V<\bigvee\left(U_{\alpha} \times V_{\alpha}\right)$ if every point of $U \times V$ is in some $U_{\alpha} \times V_{\alpha}$. Now $U$ is the union of open sets $B_{\beta}$ bounded in $U$. For each such $B_{\beta}$ and each $y \in V$ we are given that those $U_{\alpha}$ such that $y \in V_{\alpha}$ cover $U$; so finitely many of them cover $B_{\beta}$. The intersection of the corresponding $V_{\alpha}$ is a neighborhood $N_{y}$ of $y$; glancing at $X \times N_{y} \subset X \times Y$, we see that $B_{\beta} \times N_{y} \leqslant \bigvee\left(U_{\alpha} \times V_{\alpha}\right)$. Then in the same way we get $B_{\beta} \times V \leqslant \bigvee\left(U_{\alpha} \times V_{\alpha}\right)$. A third such step gives $U \times V \leqslant \bigvee\left(U_{\alpha} \times V_{\alpha}\right)$.

THEOREM 2. If two subspaces of a Hausdorff space have no common point but a nonempty locale intersection, then their product locale is not a space.

Proof. Let $A$ and $B$ be such subspaces of $X$. The traces on $A \times B$ of the open rectangles $U_{\alpha} \times V_{\alpha}$ of $X \times X$ disjoint from the diagonal $D$ cover the points of $A \times B$. But if $I$ is the intersection locale $A \cap B$, the diagonal of $I \times I$ is contained in $A \times B$ and (in $\left.D^{-}\right)$disjoint from $\bigvee\left(U_{\alpha} \times V_{\alpha}\right)=W$. Thus $W \cap(A \times B)$ is an open proper part of $A \times B$ containing all of the points.

THEOREM 3. A completely regular space $X$ has its locale product with every space a space if and only if $X$ is a complemented sublocale of a compact space. Then $X$ is complemented in every Hausdorff extension.

Proof. "If": When a locale product $Z \times A$ is a space and $X$ is a complemented sublocale of $Z, X \times A$ is also a space [4, 2.10]. The rest: If $X$ is noncomplemented in a compactification, or even in some Hausdorff extension $Z$ of $X$, let $Y$ be the subspace of $Z$ on the points not in $X$. In the sublocales of $Z, X \vee Y$ contains all the points, so $X \vee Y=1$. Then $X \wedge Y \neq 0$, and Theorem 2 applies.

LEMMA 1. If two open parts of a locale $X$ agree on certain sublocales $S_{i}$, they agree on the sublocale join of the $S_{i}$.

Proof. Open parts $u, v$ are complemented sublocales, so 1 in the lattice of sublocales partitions into $u \Delta v=\left(u \wedge v^{\prime}\right) \vee\left(u^{\prime} \wedge v\right)$ and its complement " $u=$ $v$ ". And $u$ and $v$ agree on $S_{i}$ if and only if $S_{i}<$ " $u=v$ ".

Lemma 2. For spaces $X, Y, X \times Y$ is a space if $X$ is the union of finitely many subspaces $S_{i}$ such that each $S_{i} \times Y$ is a space.

Proof. First, the sublocale join of the $S_{i}$ is a sublocale of $X$ containing all the points; thus it is 1 . Now observe that coordinate projection $\pi: X \times Y \rightarrow X$ associates to each sublocale $S$ of $X$ an "inverse image" $I(\pi)(S)$ which is $S \times Y$; for sublocales $S \rightarrow X$ are by definition equalizers of diagrams $X \rightrightarrows Z$, and $I(\pi)$ just takes $S$ to the equalizer of $X \times Y \rightarrow X \rightrightarrows Z$, which inspection of diagrams shows to be $S \times Y$. Finally, $I(\pi)$ preserves finite joins $[4,1.4]$. 
THEOREM 4. $A$ space $X$ has its locale product with every space a space if every nonempty closed subset $H$ of $X$ has a point with a quasi-locally compact neighborhood in $H$. For completely regular $X$, the converse is also true.

REMARK. The last sentence follows from Theorem 2 by a remark in [4, p. 23]; but the proof is not given there, and here it will not take three lines.

Proof. We use transfinite induction based mainly on Lemma 2. At limit ordinals we want, again from 2.10 of [4], the similar result for infinite families $\left\{S_{i}\right\}$ whose interiors cover $X$. Let $X_{0}=X, X_{\alpha+1}=$ the set of all points of $X_{\alpha}$ having no quasi-locally compact neighborhood in $X_{\alpha}$; for limit $\lambda, X_{\lambda}=\cap\left[X_{\alpha}: \alpha<\lambda\right]$. (This intersection is the sublocale infimum since all $X_{\alpha}$ are closed.) As long as $X_{\alpha}$ is nonempty, it is locally quasi-locally compact somewhere and $X_{\alpha+1}$ is a proper subset; so finally $X_{\beta}$ is empty. Observe, for $U$ open in $X, U_{1}=X_{1} \cap U$, and inductively $U_{\alpha}=X_{\alpha} \cap U$.

If $X_{1}$ is empty, the result is Theorem 1. Suppose it holds for all $Y$ with $Y_{\alpha}$ empty, and consider $X$ with $X_{\alpha}$ nonempty but quasi-locally compact ( $X_{\alpha+1}$ empty). Lemma 2 applies to closed $X_{\alpha}$ and its complement $U$. Again, at a limit ordinal $\lambda$ with $X_{\lambda}$ empty the open complements of $X_{\alpha}(\alpha<\lambda)$ cover $X$; and "if" is proved.

If completely regular $X$ has a nonempty closed set $H$ locally compact nowhere, then in $\beta X$ both $X$ and the subspace on the points of $\beta X-X$ contain the smallest dense sublocale of $\mathrm{H}^{-}$; by Theorem 2, their product is not a space.

\section{REFERENCES}

1. J. Benabou, Treillis locaux et paratopologies, Séminaire C. Ehresmann, 1957/58, Fac. des Sciences de Paris, 1959.

2. C. H. Dowker and D. Strauss, Sums in the category of frames, Houston J. Math. 3 (1977), 17-32.

3. K. H. Hofmann and J. D. Lawson, Irreducibility and generation in continuous lattices, Semigroup Forum 13 (1977), 307-353.

4. J. R. Isbell, Atomless parts of spaces, Math. Scand. 31 (1972), 5-32.

5. Z. Shmuely, The structure of Galois connections, Pacific J. Math. 54 (1974), 209-225.

Department of Mathematics, State University of New York, Buffalo, New York 14214 